\title{
OPTIMAL ILLUSION OF CONTROL AND RELATED PERCEPTION BIASES
}

\author{
Olivier Gossner \\ Jakub Steiner
}

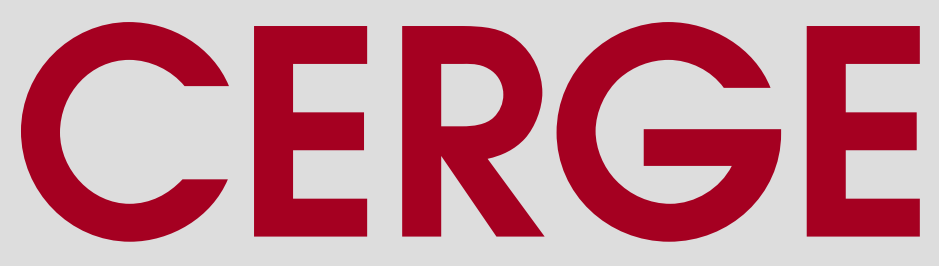




\title{
Working Paper Series (ISSN 1211-3298)
}

\section{Optimal Illusion of Control and Related Perception Biases}

\author{
Olivier Gossner \\ Jakub Steiner
}

CERGE-EI

Prague, September 2016 
ISBN 978-80-7343-378-9 (Univerzita Karlova v Praze, Centrum pro ekonomický výzkum a doktorské studium)

ISBN 978-80-7344-394-8 (Národohospodářský ústav AV ČR, v. v. i.) 


\title{
Optimal Illusion of Control and Related Perception Biases*
}

\author{
Olivier Gossner ${ }^{\dagger}$ \\ CREST, CNRS - École Polytechnique, Université Paris Saclay \\ and London School of Economics \\ Jakub Steiner ${ }^{\ddagger}$ \\ University of Edinburgh and Cerge-Ei
}

September 2016

\begin{abstract}
We study perception biases arising under second-best perception strategies. An agent correctly observes a parameter that is payoff-relevant in many decision problems that she encounters in her environment but is unable to retain all the information until her decision. A designer of the decision process chooses a perception strategy that determines the distribution of the perception errors. If some information loss is unavoidable due to cognition constraints, then (under additional conditions) the optimal perception strategy exhibits the illusion of control, overconfidence, and optimism.
\end{abstract}

\footnotetext{
${ }^{*}$ We thank Andrew Clausen, Bard Harstad, Yuval Heller, Ed Hopkins, Drazen Prelec, József Sákovics, Colin Stewart, Andy Zapechelnyuk, and the audiences at YEM 2016, BRIC 2016, GAMES 2016 and the Limited Cognition workshop in Barcelona for comments. Ludmila Matysková has provided excellent research assistance and Judith Levi has carefully edited the text.

${ }^{\dagger}$ email: olivier.gossner@polytechnique.edu

${ }^{\ddagger}$ email: jakub.steiner@ed.ac.uk
} 


\section{Introduction}

Within economic discourse, the idea that the human mind is imperfect and that some information is necessarily lost during any decision process dates back at least to Simon (1955). Under this premise, an agent faces, apart from the standard action choice, a problem of lossy data compression; she must choose which information to retain and which to let go. Within information theory, the problem of lossy data compression is defined by (i) the information processing technology that determines feasible perception error distributions and (ii) the loss function that specifies the loss from each possible misperception of the processed parameter. ${ }^{1}$ Information theory mainly focuses on the former and studies sophisticated usage of limited information channels, minimizing simple ad hoc loss functions. We approach the problem from the economic perspective and focus on the microfoundation of the loss function.

For instance, the agent may not be able to perfectly retain all relevant information about the probability of her having more or less talent and thus may arrive at the action choice as under- or overconfident about her own talent. We provide a definition of the loss associated with each such misperception that builds on the standard economic welfare approach. In our model, an agent maximizes a random utility that depends on the chosen action from a fixed action set and the agent's type. Each realization of the random utility function represents a decision problem that the agent may encounter and the distribution of the utility function provides a statistical description of her environment. The misperceived probability of high talent distorts choice and leads to a welfare loss in some of the problems. We define the loss associated with this misperception as the welfare loss averaged across all decision problems in the agent's environment. We characterize the loss function, given the distribution of utility functions, and we report conditions under which the loss function exhibits useful comparative statics. In particular, we identify cases in which overconfidence generates a smaller loss than does underconfidence of a same magnitude.

Our main result relates such asymmetries of the loss function to the degree of control the agent has over her utility. We assume that the agent can be of high or low type and consider settings in which the high type has more control over her utility than the low type, in the sense that random payoff for any fixed action has a higher variance in the high state than in the low one. Then, for most realizations of the utility function, the agent's choice has a larger impact on her payoff in the high state than in the low one. We

\footnotetext{
${ }^{1}$ See Cover and Thomas (2006) for introductory reading on lossy data compression.
} 
show that, under this assumption, an overvaluation of the objective probability $p$ leads to a smaller loss than an undervaluation of the same magnitude.

The intuition is as follows. A misperception of the objective probability $p$ for a subjective probability $q$ leads to a loss only in those decision problems in which the optimal actions at $p$ and $q$ differ. Thus, the loss arises if and only if the agent is indifferent between the two actions at some intermediate probability $s$ in-between $p$ and $q$. When an agent's high type has more control than the low type, a tie between the two actions is less likely to arise in an upper neighborhood of the objective probability $p$ than in the lower neighborhood of $p$ of the same magnitude, because the random expected payoff for any given action at probability $p$ has a more spread-out density for higher values of the probability $p$. Hence, the upward error is less often consequential than its downward counterpart.

Therefore, overvaluing one's control over one's own well-being leads to a smaller loss than undervaluing one's control. When errorless perception is infeasible, optimization over the distributions of the perception errors leads to a second-best stochastic perception that is biased toward the state of the high control. The imperfect agent exhibits an illusion of control. ${ }^{2}$ The effect generates further behavioral phenomena. We provide examples in which the choice of highly talented or well-informed people has a larger impact on their well-being than the choice of less talented or less well-informed people, and show that an overconfidence bias is optimal in these cases when perfect perception is infeasible. The illusion of control can also generate optimism. An optimizing agent benefits from a high spread of the payoffs to the available actions, and hence (under additional conditions) the bias toward the high-control state coincides with the bias toward the state in which the optimizing agent fares better.

Although cognition constraints are uncontroversial, little is known about their exact form. We examine three variants of the perception problem that differ in the set of the feasible stochastic perception strategies. In the first and simplest version, the agent observes the true probability $p$ of the high state, chooses to memorize a probability $m$, and recalls probability $q=m+\varepsilon$, where $\varepsilon$ is a small additive error of zero mean. We show that when the agent has more control in the high state than in the low one, the optimal perception strategy is biased upwards. In this case, the agent memorizes a probability $m$ that exceeds the true probability $p$, and the recalled probability $q$ exceeds $p$ on average

\footnotetext{
${ }^{2}$ Illusion of control refers to the overestimation of one's ability to impact one's own well-being. As an extreme example, casino visitors may overestimate the relevance of their choices over equivalent lotteries. The term was introduced by Langer (1975).
} 
across the error $\varepsilon$. The agent in this setting is naive in the sense that she does not form Bayesian posteriors about the objective probability $p$ upon recalling $q$; instead, she treats the subjective probability $q$ as if it were accurate. Such a model can capture an agent who makes mistakes in evaluating the available evidence and arrives at a perturbed posterior $q$ instead of forming the correct posterior $p$. The optimal perception strategy optimizes the distribution of the updating errors.

In the second version of the perception problem, we consider a sophisticated agent who loses information during the decision process but rationally utilizes all the information she retains. The agent has a binary memory and thus forms a binary partition of the probability interval, remembers the cell of the partition into which the objective probability $p$ belongs, and forms a Bayesian posterior about $p$ at the decision stage. We show that, if the prior density of $p$ is symmetric around $1 / 2$ and the agent has more control in the high state than in the low one, then the upper cell of the optimal partition is larger than the lower cell. Thus, the agent is more likely to hold a belief that attaches a high probability to the high type than a belief favoring the low type.

Our third specification of the perception problem generalizes and unifies the previous two settings. We assume that the agent chooses from a set of available stochastic perceptions, where each such perception is a random variable. The agent observes the objective probability $p$, chooses a stochastic perception of the probability $p$ from the available set, forgets $p$, and forms a perception equal to the realization of the chosen random variable. The perception problem is to optimize over the perception strategies that map from $p$ to the set of stochastic perceptions. We assume that the menu of available stochastic perceptions is ordered with respect to first-order stochastic dominance and that the agent has more control in the high state than in the low one.

We compare solutions of two perception problems, both of which share the same menu of the stochastic perceptions but differ in the loss functions. In the precision maximization problem, the agent minimizes the mean-square error of her perception. In the payoff maximization problem, the agent minimizes the welfare loss. We use minimization of mean-square error as a benchmark because it is a widespread criterion in statistics and in information theory, even though it ignores asymmetries of the welfare losses from misperceptions. Comparison of the two solutions isolates the impact of the asymmetries on the payoff-maximizing perception. We show that, compared to the precision-maximizing benchmark, the payoff-maximizing strategy is biased upwards: the payoff-maximizing per- 
ception at each objective probability $p$ stochastically dominates the precision-maximizing perception. Thus, an outsider observing the joint density of the objective and subjective probabilities and who conjectures that the agent is maximizing precision will conclude that the agent is biased toward the high control state.

As a technical contribution, we provide a simple and intuitive formula that, for a given distribution of the utility functions, characterizes the loss from misperceiving the objective probability $p$ as $q$. This characterization shows that the loss function can be expressed as an integral formula that depends only on the value function. The value function, for each probability $p$ of the high state, specifies the payoff of the optimizing agent who has correct perception, averaged out across all the decision problems in her environment. The value function has a simple analytical form when the utility function has a Gaussian distribution, and hence we focus on the Gaussian payoffs in most of the paper. We obtain the asymmetry of the loss function and the bias of the optimal perception in settings in which (i) the payoff for each action consists of two additive terms, and (ii) one of the terms has a relatively higher weight for the agent's high type than for the low type. We extend the results beyond the Gaussian payoff distributions in the last section of the paper.

Rate-distortion theory is a branch of information theory originated by Shannon (1948, 1959). It studies optimal communication via a noisy channel. One of the primitives of the theory is an exogenous map that specifies a loss to each input and output of the communication. A popular loss function is the square error. In our paper, the loss function is derived from the agent's environment and is equal to the average welfare loss caused by the distortion. ${ }^{3}$

Once we establish the relevant loss function, we let the agent engage in optimal error management: she avoids the costlier types of the errors. Beyond information theory, the idea of error management has been studied in several scientific disciplines such as biology and psychology. In Haselton and Buss (2000), males' interpretation of females' sexual interest is biased toward false-positives because false-negatives have a larger fitness cost. Nettle (2004) provides a foundation for optimism by arguing for asymmetry of the optimistic and pessimistic error costs. See Johnson et al. (2013) for an interdisciplinary literature review. We contribute to this literature a formal characterization of loss from misperception based on a statistical description of the agent's environment.

Sims $(1998,2003)$ has introduced information capacity constraints to economics. The

\footnotetext{
${ }^{3} \mathrm{An}$ appropriate definition of the loss function is a nontrivial and important part of the data compression problem. See the discussion of the loss function for compression of images in Wang et al. (2004).
} 
subsequent literature on rational inattention studies the information acquisition and processing of an agent who, as in our model, is unsure about a payoff-relevant parameter but who, unlike our agent, knows her payoff function. In that setting (assuming signal cost is nondecreasing in Blackwell informativeness), the agent acquires only an action recommendation and no additional information beyond that needed for choice. In our model, the agent does not know her payoff function when she processes information and thus forms beliefs beyond those needed for the mere action choice in any given problem. The resulting optimal information structure can then be naturally interpreted as a perception of the payoff parameter. ${ }^{4}$

Our assumption that the perception strategy is optimized across many decision problems has appeared in the literature on the evolution of the utility functions. This literature studies performance of decision processes across a distribution of the fitness rewards to the available actions. Depending on the constraints assumed, the optimal decision criterion is either the expected utility maximization, as in Robson (2001), or its behavioral variants, as in Rayo and Becker (2007) and Netzer (2009). (See Robson and Samuelson (2010) for a literature review.) Most of this literature focuses on choice under certainty. Two exceptions who, like us, focus on the probability perception are Herold and Netzer (2010), who propose that biases in probability perception serve as a correction of another behavioral distortion; and Compte and Postlewaite (2012), who conclude that an agent with limited memory benefits from ignoring weakly informative signals.

We contribute to the branch of the bounded rationality literature that emphasizes limited memory. Mullainathan (2002) studies behavioral implications of exogenous imperfect memory usage. Like us, Dow (1991) and Wilson (2014) study behavioral implications of an agent who optimizes her usage of limited memory, but they examine effects that are different from ours. In his survey, Lipman (1995) focuses on memory-related frictions.

This paper adapts the framework from Steiner and Stewart (2016) to new behavioral phenomena. A subtle but important difference in settings between the two papers is that Steiner and Stewart analyze perception of a probability that idiosyncratically affects the payoff to only one action, whereas this paper studies perception of a probability that affects payoffs to all actions. Because of this difference, the two papers arrive at opposite results. Steiner and Stewart derive the optimal overvaluation of small probabilities and offer an explanation for prospect-theory probability weighting. This paper, on the other hand,

\footnotetext{
${ }^{4}$ Within the rational inattention literature, Woodford (2012a,b) studies behavioral biases resulting from perception frictions; in these studies he assumes the mean square error loss criterion.
} 
shows that the agent is overconfident about the precision of her belief and thus undervalues small probabilities attached to unlikely outcomes; it thus provides a microfoundation for the illusion of control and for overconfidence. In addition to the new application, the current paper makes a technical advance. The loss characterization based on the value function is novel and allows us to solve settings with large action sets.

Overconfidence, optimism, and other perception biases impact choices in personal, economic and political life. ${ }^{5}$ In Brunnermeier and Parker (2005), overconfidence bias arises because people derive hedonic payoffs from positive beliefs; in Bénabou and Tirole (2002) confidence helps to resolve an intrapersonal conflict caused by time-inconsistent preferences, while in Compte and Postlewaite (2004), confidence is an explicit production factor. Johnson and Fowler (2011) show that overconfidence provides a strategic advantage in a conflict. In our model, confidence has no intrinsic value and is a feature of the least costly feasible distribution of the perception errors.

\section{Loss from Misperception}

An agent faces a randomly drawn decision problem. She chooses an action $a \in A=$ $\{1, \ldots, n\}, n \geq 2$, and receives payoff $u(a, \theta)$ where $\theta \in\{l, h\}$ is a payoff state. (Below we use the terms payoff state and payoff type interchangeably.) The agent's perception of the probability distribution over $\theta$ is imperfect. Correspondingly, we distinguish between an objective probability $p$ and a subjective probability $q$ of the state $h$. The objective and subjective probabilities of the state $l$ are $1-p$ and $1-q$ respectively. The expected payoff for action $a$ under the probability $p$ is $u(a, p)=p u(a, h)+(1-p) u(a, l)$.

We study a two-stage decision process in which a perception stage is followed by a choice stage. In the choice stage, the agent knows the realized payoff function $u$, mistakenly perceives the true probability $p$ as $q$, chooses an action

$$
a_{q, u}^{*}=\arg \max _{a \in A} u(a, q)
$$

that maximizes her perceived payoff, and receives the true payoff $u\left(a_{q, u}^{*}, p\right){ }^{6}$

The decision problem is uncertain in the preceding perception stage. Accordingly,

\footnotetext{
${ }^{5}$ See empirical evidence for each of these domains in Puri and Robinson (2007), Hirshleifer (2001), and Ortoleva and Snowberg (2015).

${ }^{6}$ In our framework, the maximizer is unique with probability one. We therefore ignore cases with multiple optima.
} 
$u=(u(a, \theta))_{a, \theta}$ and $p$ are random variables with supports $\mathbf{R}^{2 n}$ and $[0,1]$, respectively, both admitting atomless density; $u$ and $p$ are independent. We let

$$
V(p, q)=\mathrm{E}_{u} u\left(a_{q, u}^{*}, p\right)
$$

denote the payoff of the optimizing agent who misperceives the objective probability $p$ as $q$, averaged out across the draws of the payoff function $u$.

Later in the paper, we optimize across the distributions of the perception errors. For that reason, we need to compare losses from misperception across various perceived probabilities $q$. Let the value function $v(p)=V(p, p)$ denote the expected payoff of the optimizing agent who perceives the objective probability $p$ correctly, and let

$$
L(p, q)=v(p)-V(p, q)
$$

be the expected loss due to misperceiving the true probability $p$ as $q$.

Our aim is to characterize how the distribution of the payoffs $u$ translates into the loss function $L$. To start, the next section reviews three examples that share a particular property of the payoff distribution. In all of them, for typical draws of $u$, the choice of the agent in the high state has more impact on her well-being than her choice in the low state. We show that for such payoff distributions, a downward perception error is costlier than an upward error of the same magnitude.

\section{$2.1 \quad$ Applications}

Example 1 (overconfidence). In this application, the agent is uncertain about her talent, and the choices of highly talented individuals have on average more impact on their wellbeing than the choices of the less talented.

The agent chooses one out of $n$ projects. Project $a \in\{1, \ldots, n\}$ yields a payoff $u(a, \theta)=$ $\tau_{\theta} b_{a}-c_{a}$, where $c_{a}$ is the participation cost, $\tau_{\theta}>0$ is the agent's talent in state $\theta$, and $b_{a}$ is the talent-unadjusted benefit of participating in project $a$. At the choice stage, the agent observes the $2 n$ parameters $\left(b_{a}, c_{a}\right)_{a}$, attributes probability $q$ to the high state, and chooses the project that maximizes $u(a, q)=q u(a, h)+(1-q) u(a, l)$. The parameters $b_{a}$ and $c_{a}$ are independently drawn from a normal distribution at the ex ante stage.

We assume that $\tau_{h}>\tau_{l}$. Thus, for high types in comparison to low ones, benefit 
parameters $b_{a}$ are relatively more important than the $\operatorname{costs} c_{a} \cdot{ }^{7}$ Misperception of talent leads to misinformed choice and a loss for some realizations of the projects' parameters. We show below that the loss function is asymmetric in favor of the upward errors. Once we introduce optimization over the error distributions, this asymmetry will imply that when some misperception is unavoidable, the optimal perception exhibits overconfidence.

Example 2 (overprecision). In this example, the agent bets on an event. The agent may be either well informed or less informed about the event. On average, the better informed type of the agent faces larger payoff differences among the available bets than the less informed one.

The agent chooses one out of $n$ binary bets. Bet $a \in\{1, \ldots, n\}$ pays reward $r_{\gamma}^{a}$ in outcome $\gamma \in\{t, d\}$, and the probabilities of the outcomes $t$ and $d$ in state $\theta \in\{h, l\}$ are $\pi_{\theta}$ and $1-\pi_{\theta}$, respectively. Thus, the value of bet $a$ in state $\theta$ is $u(a, \theta)=\pi_{\theta} r_{t}^{a}+\left(1-\pi_{\theta}\right) r_{d}^{a}$. The agent observes the $2 n$ realized rewards $\left(r_{\gamma}^{a}\right)_{a, \gamma}$ at the choice stage. She is uncertain about the state $\theta$, and thus faces compound lotteries. The agent attributes probability $q$ to state $h$ and chooses the project that maximizes $u(a, q)=q u(a, h)+(1-q) u(a, l)$. All rewards $r_{\gamma}^{a}$ are iid. from a normal distribution at the ex ante stage.

We assume that $\pi_{h}>\pi_{l}>1 / 2$ to reflect the fact the agent is more informed about the outcome of the bet in the high state than in the low one. ${ }^{8}$ Misperception of $\theta$ leads to a mistaken belief about the outcome of the bet and to a suboptimal choice. We show below that being underconfident about one's own knowledge of the payoff-relevant outcome is relatively costlier than the opposite error and that the second-best perception strategy exhibits overprecision. ${ }^{9}$

Example 3 (illusion of control). In this example, the two states differ in the predictability of the payoff consequences of the agent's choice. The payoffs are more predictable in the high state than in the low one, and the agent's choice has on average more impact on her expected utility in the high state than in the low one.

The agent's payoff for action $a \in\{1, \ldots, n\}$ is $e_{a}+l_{a}$, where $e_{a}$ is a predictable payoff term (capturing, for instance, the predictable benefit of effort) and $l_{a}$ is a luck term. At the choice stage, the agent observes, for each $a$, the predictable term $e_{a}$ and a noisy signal

\footnotetext{
${ }^{7}$ The optimal perception bias would be the same if we would assume that (i) both types receive the same participation benefits and (ii) the participation cost of the high types is larger than that of the low types, reflecting higher opportunity costs.

${ }^{8}$ The result is identical if $\pi_{h}<\pi_{l}<1 / 2$.

${ }^{9}$ We follow here the terminology from Moore and Healy (2008), who refer to overvaluation of one's performance as "overconfidence" and overvaluation of the precision of one's belief as "overprecision".
} 
$x_{a}=l_{a}+\varepsilon_{a}$ about the luck term, but not $l_{a}$ itself. The luck terms $l_{a}$ are drawn from $\mathcal{N}(y, 1)$ and the errors $\varepsilon_{a}$ from $\mathcal{N}\left(0,1 / \gamma_{\theta}\right)$, where precision $\gamma_{\theta}$ represents the predictability of luck in state $\theta \in\{l, h\}$. Thus, the expectation over the luck term $l_{a}$ upon observing signal $x_{a}$ in the state $\theta$ is $\frac{y+\gamma_{\theta} x_{a}}{1+\gamma_{\theta}}$, and the expected payoff for action $a$ in state $\theta$ is $u(a, \theta)=e_{a}+\frac{y+\gamma_{\theta} x_{a}}{1+\gamma_{\theta}}$. The agent observes the $2 n$ parameters $\left(e_{a}, x_{a}\right)_{a}$ at the choice stage, attributes probability $q$ to state $h$, and chooses the action that maximizes $u(a, q)=q u(a, h)+(1-q) u(a, l)$. The terms $e_{a}$ are independently drawn from a normal distribution at the ex ante stage.

We assume $\gamma_{h}>\gamma_{l}$ to express that luck is less predictable in the low state than in the high state. Misperception of $p$ distorts the weight that the agent assigns to the signal $x_{a}$, and thus it distorts choice. We show that, again, the loss function is asymmetric in favor of the upward errors and the second-best perception strategy overestimates predictability of the payoffs, and thus also overestimates the impact of the agent's choice.

Proposition 1. In all three examples, the downward perception error is costlier than the upward error of the same magnitude:

$$
L(p, p-\sigma)>L(p, p+\sigma),
$$

for any $\sigma \in(0,1 / 2)$ and $p \in[\sigma, 1-\sigma]$.

The proposition follows from a general result in Corollary 1.

\subsection{Intuition}

Misperception of probability $p$ as $q$ causes a loss only when the optimal actions under the two probabilities differ: $a_{p, u}^{*} \neq a_{q, u}^{*}$. In this case, there exists a probability $s$ in-between $p$ and $q$ such that at $s$, the agent is indifferent between $a_{p, u}^{*}$ and $a_{q, u}^{*}$. Thus, misperception of $p$ as $q$ is costly for those draws of the payoffs $u$ under which a tie arises in-between $p$ and $q$. In all three examples, the variance of the payoff $u(a, p)$ is increasing in $p$. In the first example, this is because the high talent magnifies the variance of the benefit parameter $b_{a}$. In the second example, the variance of the bet value $\pi r_{t}^{a}+(1-\pi) r_{d}^{a}$ is $\pi^{2}+(1-\pi)^{2}$, which increases with $\pi$ on $(1 / 2,1)$. Thus, the more the agent knows about the outcome of the bet, the larger the payoff differences between the bet values tend to be. In the third example, the payoff consequences of each action are more predictable in the high state than in the low one, and thus the expected payoff differences are typically larger in the high state. 
Larger values of the variance of $u(a, s)$ correspond to lower likelihoods of a tie between two actions at probability $s$. Consider these two subjective perceptions: $q=p+\sigma$ and $q=p-\sigma$. Due to the decreasing payoff variance, a tie is less likely to arise at some $s$ in $(p, p+\sigma)$ than for $s \in(p-\sigma, p)$. Therefore, the upward perception error leads to a loss less often than the downward error of the same magnitude. The next section completes the above argument by incorporating into the computation the size of the loss from misperception alongside the probability of the loss.

The technical property unifying all three examples is that the payoff function can be decomposed as

$$
u(a, \theta)=u_{a}+\tau_{\theta} \eta_{a},
$$

where $u_{a}$ and $\eta_{a}$ are independent random variables, iid. across the actions, and $\tau_{h}>\tau_{l}>0$; see the appendix for this decomposition. When these conditions hold, we say that the agent has more control (over her payoff) in the high state than in the low one.

\section{Loss Characterization}

Recall that $v(p)=\mathrm{E}_{u} \max _{a} u(a, p)$ is the value of the optimizing agent with the correct perception, averaged out across the draws of the payoffs. Our main technical insight characterizes the loss function in terms of the value function.

Lemma 1. The expected payoff loss due to misperceiving the probability $p$ as $q$ is

$$
L(p, q)=\int_{p}^{q} v^{\prime \prime}(s)(s-p) d s .
$$

We illustrate Lemma 1 in Figure 1. The left graph depicts functions $u(a, p)$ for all actions $a$ under a particular realization of the payoffs $u$. The agent with perception $q$ chooses the action associated with the full line, whereas the optimal choice at the objective probability $p$ is the action associated with the dotted line, which implies the loss $L(p, q ; u)$. When we let $v(p ; u)=\max _{a} u(a, p)$, the loss is the error of the linear approximation of the realized value function $v(p ; u)$ when it is approximated at $q$. The right graph depicts the expected loss as the error of the linear approximation of the expected value function.

Proof of Lemma 1. Note that $v(p)=\max _{q} V(p, q)=V(p, p)$. Applying the envelope theorem to

$$
V(p, q)=\mathrm{E}_{u}\left[(1-p) u\left(a_{q, u}^{*}, l\right)+p u\left(a_{q, u}^{*}, h\right)\right]
$$



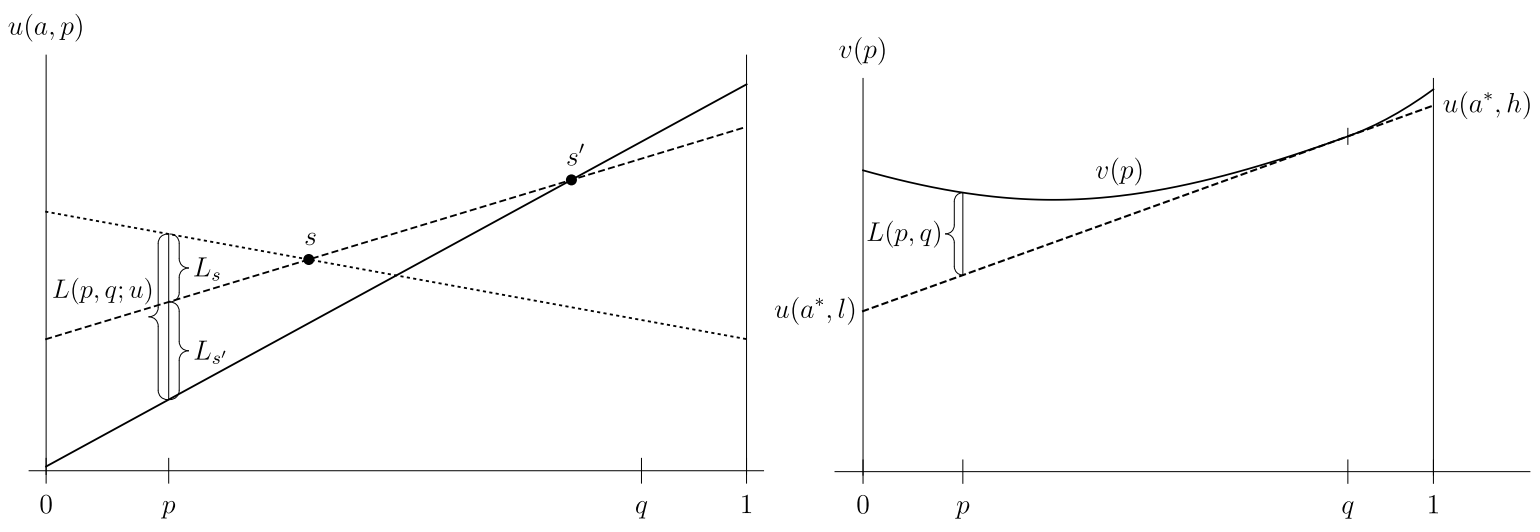

Figure 1: Left graph: loss from misperceiving $p$ as $q$ for a realized utility function. Right graph: Ex ante expected loss from misperceiving $p$ as $q$.

gives

$$
v^{\prime}(p)=\left.\frac{\partial}{\partial p} V(p, q)\right|_{q=p}=\mathrm{E}_{u}\left[u\left(a_{p, u}^{*}, h\right)-u\left(a_{p, u}^{*}, l\right)\right] .
$$

Using the linearity of the expected payoff with respect to probability and (3), we obtain

$$
V(p, q)=v(q)-v^{\prime}(q)(q-p)
$$

and thus,

$$
L(p, q)=v(p)-V(p, q)=v(p)-v(q)+v^{\prime}(q)(q-p)
$$

is the error of the linear approximation of the value function $v(p)$ when it is approximated at $q$. Expression (2) follows once we integrate by parts:

$$
\int_{p}^{q} v^{\prime \prime}(s)(s-p) d s=\int_{p}^{q} v^{\prime}(s) d s-\left[\left(v^{\prime}(q)-v^{\prime}(s)\right)(s-p)\right]_{p}^{q}=L(p, q) .
$$

\subsection{The role of $v^{\prime \prime}$}

The second derivative of the value function plays a central role in our analysis. We offer intuitive explanations of how $v^{\prime \prime}$ is related to losses and to the likelihood of suboptimal choices.

Consider an agent with a prior belief $p$ who receives a weakly informative signal upon which she updates her belief to $\pi$ in a neighborhood of $p$. When the Bayesian constraint $\mathrm{E} \pi=p$ and the second-order Taylor expansion are used, the value of the received infor- 
mation is

$$
\mathrm{E} v(\pi)-v(p) \approx v^{\prime \prime}(p) \operatorname{Var}(\pi) / 2
$$

where the information value results from the action adjustment to the updated belief.

We point out a geometric representation of the loss from a small misperception that highlights the fact that the loss arises due to distortion of choice. Notice in the left graph of Figure 1 that when the realized value function $v(\cdot ; u)$ is linear in the neighborhood of $p$, the optimal action is identical for all the probabilities near $p$; hence, a small misperception is inconsequential. A small misperception of the true probability $p$ leads to a loss only if the realized value function exhibits a kink in a neighborhood of $p$ where the kink represents an action reversal.

Now consider potentially large perception errors. $L(p, q ; u)$ is positive when the optimal choices at $p$ and $q$ differ, and hence the loss is positive when the agent is indifferent between the first-best and the second-best action at some $s$ between $p$ and $q$. Figure 1 illustrates a situation in which such indifference arises at two values $s$ and $s^{\prime}$ between $p$ and $q$. The figure shows that, in this case, the loss $L(p, q ; u)$ is the sum of two parts, $L_{s}$ and $L_{s^{\prime}}$, where each of these loss contributions is identified with a tie arising at probabilities $s$ and $s^{\prime}$. The ex ante expected loss $L(p, q)$ can thus be expressed as an integral $\int_{p}^{q} w(s)(s-p) d s$, where the weight $w(s)$ captures the likelihood of the tie at $s$ and the angle of the intersection of the graphs of $u(a, p)$ for the two best actions at $s$. According to the lemma, the weight $w(s)$ equals the second derivative of the value function. Indeed, $v^{\prime}(p ; u)$ differs across the left and the right neighborhoods of $s$ if and only if there is a tie at $s$. Thus, $v^{\prime \prime}(s)$ is the likelihood of the tie among the two best actions at $s$, modified by the angle of the intersection. We formalize this idea in Lemma 4 in section 6 .

\subsection{Gaussian Payoff Distributions}

We show here that the value function has a simple analytical form and the loss characterization becomes tractable when the payoff distribution is Gaussian. We address general distributions in section 6 .

We assume in this section that the draws of the payoff values are independent across the actions $a$; however, for each $a$, we allow for a correlation of the payoffs $u(a, l)$ and $u(a, h)$ across the two states. The payoff pair $(u(a, l), u(a, h))$ is drawn from a bivariate normal distribution $\mathcal{N}(\mu, \Sigma)$, where $\mu=\left(\mu_{l}, \mu_{h}\right)$ is the vector of means and $\Sigma=\left(\Sigma_{\theta, \theta^{\prime}}\right)_{\theta, \theta^{\prime}}$ 
is the covariance matrix. The covariance allows us to accommodate applications in which the payoffs in the two states are generated by related processes, making the misperceptions natural.

The value function for the Gaussian setting is

$$
v(p)=(1-p) \mu_{l}+p \mu_{h}+c \sqrt{(1-p)^{2} \Sigma_{l l}+2 p(1-p) \Sigma_{l h}+p^{2} \Sigma_{h h}},
$$

where the constant $c>0$ is the expectation of the maximum of $n$ independent draws from the standard normal distribution. To see this, note that the payoffs $u(a, p)$ are iid. from $\mathcal{N}\left((1-p) \mu_{l}+p \mu_{h},(1-p)^{2} \Sigma_{l l}+2 p(1-p) \Sigma_{l h}+p^{2} \Sigma_{h h}\right)$. Result (5) then follows from the scale and translation invariances of the normal distribution: the expectation of the maximum of $n$ iid. draws from $\mathcal{N}\left(\nu, \omega^{2}\right)$ is $\nu+c \omega$. The explicit expression for the value function allows us to characterize the loss function in any Gaussian setting. We focus on the settings in which the agent has more control (over her payoff) in the high state than in the low one; see (1).

When the agent has more control in the high state than in the low one, ties are relatively likely at low probabilities $p$. Hence, a small misperception of low probabilities leads relatively often to a choice distortion, and to a relatively large welfare loss.

Lemma 2. If the agent has more control in the high state than in the low one and the payoffs are normally distributed, then $v^{\prime \prime}(p)$ is decreasing.

The next corollary follows from Lemmas 1 and 2 . It summarizes the results so far and applies to all examples in section 2.1.

Corollary 1 (asymmetric losses). If the agent has more control in the high state than in the low one and the payoffs are normally distributed, then a downward perception error is costlier than an upward error of the same magnitude: $L(p, p-\sigma)>L(p, p+\sigma)$, for any $\sigma \in(0,1 / 2)$ and all $p \in[\sigma, 1-\sigma]$.

\section{Second-Best Perception}

The first-best perception strategy - maximizing the expected payoff- sets $q=p$ for all $p$. We assume that perception is constrained to be noisy and we study second-best perception strategies. 
We consider three specifications of the perception problem that differ in the constraints on the design of the decision process. We view the first specification as the simplest setting that allows us to discuss the second-best perception. The second setting relaxes the agent's naiveté and the third setting generalizes and unifies.

\subsection{A Naive Perception Problem}

To model the noise, a perception strategy associates to each objective probability $p$ a distribution over the subjective perceptions $q$. In this section, a strategy assigns a stimulus $m(p)$ to each objective probability $p \in[0,1]$, and a stimulus $m$ triggers a perception $q=$ $m+\varepsilon$, where $\varepsilon$ is a binary random variable taking values $\sigma$ and $-\sigma$ with equal probabilities, where $\sigma \in(0,1 / 2)$. Thus, a perception strategy is a map $m:[0,1] \longrightarrow[\sigma, 1-\sigma]{ }^{10}$

A particular example of a perception strategy is the unbiased perception $m(p)=p$ for $p \in[\sigma, 1-\sigma]$. This strategy does not exhibit a systematic error since $\mathrm{E}[q \mid p]=p$ for all $p \in[\sigma, 1-\sigma]$. The unbiased strategy is a benchmark to which we compare the optimal perception strategy $m^{*}(p)$ that solves a naive perception problem for each $p$,

$$
m^{*}(p) \in \arg \min _{m \in[\sigma, 1-\sigma]} \mathrm{E}_{\varepsilon} L(p, m+\varepsilon) .
$$

Note that (6) is equivalent to payoff maximization.

We summarize the two-stage decision process as follows. The agent observes the objective probability $p$ and memorizes a probability $m(p)$ at the perception stage, where $m(p)$ is independent of the payoff function $u$. At the choice stage, the agent recalls probability $q=m(p)+\varepsilon$ (but does not recall $p$ and $\varepsilon$ ), and chooses an optimal action $a_{q, u}^{*}$ under her perception $q$ and payoff $u$.

Lemma 1 yields a simple first-order condition.

Proposition 2. Let $q=m^{*}(p)+\varepsilon$ be the stochastic perception under the perception strategy solving the naive perception problem (6) when the true probability is $p$. If the optimal stimulus $m^{*}(p)$ is in $(\sigma, 1-\sigma)$, then

$$
\mathrm{E}_{\varepsilon}\left[v^{\prime \prime}(q)(q-p)\right]=0 .
$$

\footnotetext{
${ }^{10}$ The simple additive error specification is not well suited for modelling perception of probabilities near the boundaries of the probability interval. See section 4.3 for a general perception technology that allows for nonadditive errors and can naturally accommodate probabilities near 0 and 1 . Here, we ensure that every perception $q$ is in the $[0,1]$ interval by restricting $m$ to $[\sigma, 1-\sigma]$.
} 
Consider a stimulus $m \in[p-\sigma, p+\sigma] .{ }^{11}$ A marginal increase in $m$ decreases the perception error $|m+\varepsilon-p|$ when $\varepsilon=-\sigma$, and increases it when $\varepsilon=\sigma$. A marginal change of the perception $q$ affects the agent's payoff only if it affects her choice - that is, when the tie arises in-between the first-best and the second-best action at $q$. The firstorder condition thus equalizes across the two perception errors the likelihood of the tie at $q=m+\varepsilon$, adjusted for the angle of the intersection and multiplied by the size of the misperception $|q-p|$.

When $v^{\prime \prime}(p)$ is monotone, the direction of the bias can be determined without fully solving for the optimal perception.

Proposition 3. If $v^{\prime \prime}(p)$ is decreasing, then the perception strategy that solves the naive perception problem (6) is biased upwards: $m^{*}(p)>p$ and $\mathrm{E}[q \mid p]>p$ for all $p \in(0,1-\sigma)$.

The next result summarizes conditions on the decision problem that generate frequent overestimation of the probability of the high-control state.

Corollary 2 (optimal illusion of control). If the agent has more control in the high state than in the low one and the payoffs are normally distributed, then the perception strategy that solves the naive perception problem (6) is biased upwards: $m^{*}(p)>p$ and $\mathrm{E}[q \mid p]>p$ for all $p \in(0,1-\sigma)$.

\subsection{A Sophisticated Perception Problem}

The agent from the previous section is naive in that she does not fully utilize the information available to her at the choice stage. This section studies the sophisticated perception strategies of an agent who loses some information during the decision process, but who is then fully capable of utilizing the information retained until the choice.

Let $M=\{0,1\}$ be a binary message space. At the perception stage, the agent observes $p$, and retains information about it according to a perception function $m(p), m:[0,1] \longrightarrow$ $M$. At the choice stage, the agent chooses an action knowing payoffs $u=(u(a, \theta))_{a, \theta}$ and remembering the message $m(p)$. Her behavior at this stage is therefore described by a choice rule $c: \mathbf{R}^{2 n} \times M \longrightarrow A$. The sophisticated perception problem is:

$$
\max _{m(\cdot), c(\cdot, \cdot)} \mathrm{E}_{u, p} u(c(u, m(p)), p) .
$$

\footnotetext{
${ }^{11}$ Stimulus $m>p+\sigma$ is dominated by $m=p+\sigma$, and $m<p-\sigma$ is dominated by $m=p-\sigma$.
} 
The next lemma states that the optimal perception function $m(\cdot)$ generates a binary partition $\mathcal{I}\left(p^{*}\right)=\left\{\left[0, p^{*}\right),\left[p^{*}, 1\right]\right\}$ of the probability interval. The agent at the choice stage remembers that $p$ belongs to $I \in \mathcal{I}\left(p^{*}\right)$, forms a Bayesian posterior $q=\mathrm{E}[p \mid p \in I]$, and chooses the optimal action $a_{q, u}^{*}$ under the posterior belief $q$.

Lemma 3. There exists $p^{*}$ such that $m(p)$ is an indicator function of one of the two intervals of the partition $\mathcal{I}\left(p^{*}\right)$ for almost all $p$; and $c(u, m)=a_{q(m), u}^{*}$ for almost all $u$, where $q(m)$ is the posterior belief $\mathrm{E}[p \mid m(p)=m]$.

Let us denote the density of $p$ by $\varphi$ and assume that it has support on $[0,1]$ and is symmetric around half: $\varphi(p)=\varphi(1-p)$ for all $p \in[0,1]$. We interpret the symmetry as a neutrality assumption abstracting from biases driven by an asymmetry of the prior $\varphi$.

Denote the two Bayesian posteriors by $\underline{q}\left(p^{*}\right)=\mathrm{E}\left[p \mid p<p^{*}\right]$, and $\bar{q}\left(p^{*}\right)=\mathrm{E}\left[p \mid p \geq p^{*}\right]$.

Proposition 4 (sophisticated perception). If $v^{\prime \prime}(p)$ is decreasing, and $\varphi(p)$ is symmetric around $1 / 2$, then the optimal threshold probability $p^{*}$ is less than $1 / 2$ and the agent attains the high posterior $\bar{q}\left(p^{*}\right)$ with a higher probability than the low posterior $\underline{q}\left(p^{*}\right)$.

As seen in the proof in the appendix, Proposition 4 holds under a weaker condition on $v^{\prime \prime}(\cdot)$, namely, when $v^{\prime \prime}(p)>v^{\prime \prime}(1-p)$ for all $p \in[0,1 / 2)$. This condition is satisfied in any Gaussian setting in which $\operatorname{Var} u(a, h)>\operatorname{Var} u(a, l)$; this inequality allows for any covariance of $u(a, l)$ and $u(a, h)$, and thus relaxes the additive structure of the payoffs from $(1)$.

Let us summarize the results for the sophisticated perception.

Corollary 3. If the agent has more control in the high state than in the low state, the payoffs are normally distributed, and $\varphi(p)$ is symmetric around $1 / 2$, then $p^{*}<1 / 2$ and the agent attains the high posterior $\bar{q}\left(p^{*}\right)$ with a higher probability than the low posterior $\underline{q}\left(p^{*}\right)$.

To obtain some intuition for the last result, recall from (4) that $v^{\prime \prime}(p)$ can be interpreted as a local value of information. Decreasing $v^{\prime \prime}(p)$ implies that the local value of information is higher in the lower half of the probability interval than in the upper one. The optimal binary partition then discriminates between the probabilities in the lower half of the probability interval at the expense of not distinguishing among the probabilities in the upper half. 
The result of this section is related to the work by Menezes et al. (1980) on preferences against increasing downside risk. The authors study preferences over wealth lotteries with a common mean, and variances that differ in the size of the downside risk. They show that agents with a positive third derivative of the utility function prefer a smaller downside risk. If we interpret $v(p)$ as a "utility" over $p$, then our agent has a negative third derivative and thus prefers the "downside risk" - her optimal partition leads to a frequent but modest upward update to $\bar{q}\left(p^{*}\right)$ and an infrequent but large update to $q\left(p^{*}\right) .{ }^{12}$

\subsection{A General Perception Problem}

In this section, we generalize results on the perception biases while being as agnostic as possible on the perception technology. Our aim is to define perception biases in a general framework and to clarify the observable predictions of the model.

To that end, we take the perspective of an outsider who observes, for each value of the objective probability $p$, the conditional density $\mu(q \mid p)$ of the perceived probabilities $q$ formed by the agent. We provide a definition of the perception bias that relies only on the observed conditional densities and makes no assumptions about the agent's perception technology. We then extend the results from the previous sections and show that the optimal perception is biased toward the high state if $v^{\prime \prime}(p)$ is decreasing and if the perception technology satisfies a regularity condition.

The benchmark against which we define the perception bias is a statistician whose sole goal is to maximize the precision of her perception, where precision is measured by the inverse of the mean-square error. As the agent's true objective is payoff maximization and not precision maximization, the agent in general exhibits a different perception from that of the statistician. Our goal is to characterize the difference between the optimal perception strategies of the agent and that of the statistician because it reflects the asymmetries of the loss function that are absent from the statistician's objective.

The following setting generalizes the two previous perception technologies. The agent observes the objective probability $p$ and chooses a stochastic perception $\mathbf{q} \in Q$, where $\mathbf{q}$ is a random variable with support $[0,1]$. For each $p$, the agent solves the general perception problem

$$
\min _{\mathbf{q} \in Q} \mathrm{E} L(p, \mathbf{q})
$$

The set $Q$ of the feasible random perceptions $\mathbf{q}$ depends on the details of the perception

\footnotetext{
${ }^{12}$ We thank Collin Raymond for pointing this connection out to us.
} 
technology available to the agent. For instance, for the technology from section 4.1, the set $Q$ contains binary lotteries which attain values $m+\sigma$ and $m-\sigma$ with equal probabilities. For the technology from section 4.2 , the set $Q$ consists of two random variables with degenerate distributions, one of which attains the value $q\left(p^{*}\right)$ with certainty and the other of which attains the value $\bar{q}\left(p^{*}\right)$. As in section 4.1 , the set $Q$ can be exogenous, or, as in section 4.2, the set $Q$ may be the outcome of an optimization process.

The outsider observes the conditional density $\mu(q \mid p)$ of the subjective probability $q$ for each objective probability $p .{ }^{13}$ When we let $\mathbf{q}(p)$ be a random variable with density $\mu(q \mid p)$, the observed data reveal that the agent can implement any stochastic perception $\mathbf{q}(\hat{p})$ for $\hat{p} \in[0,1]$. Given an objective probability $p$, a statistician whose motive is to maximize precision would choose the stochastic perception minimizing $\mathrm{E}(\mathbf{q}-p)^{2}$. Thus, under the outsider's conjecture that the observed agent is such a statistician, each $p \in[0,1]$ should belong to this set:

$$
P^{*}(p)=\arg \min _{\hat{p} \in[0,1]} \mathrm{E}(\mathbf{q}(\hat{p})-p)^{2}
$$

When $p \notin P^{*}(p)$, the agent is revealed to be biased relative to the objective of precision maximization. Unsurprisingly, when the actual objective is payoff maximization, the agent will be typically biased. The next result provides conditions under which the revealed bias will be in the upward direction.

Recall that a random variable with cdf. $F_{X}$ strictly stochastically dominates another random variable with cdf. $F_{Y}$ if $F_{X}(z) \leq F_{Y}(z)$ for every $z$, and the inequality is strict for some $z$.

Proposition 5. Assume that the set $Q$ of the feasible perceptions is ordered with respect to the strict stochastic dominance order, that $v^{\prime \prime}(p)$ is decreasing, and that $\mathbf{q}(p)$ solves the problem (9). If the agent is revealed to be biased at $p$, that is, $p \notin P^{*}(p)$, then $\sup P^{*}(p)<p$ and $\mathbf{q}(p)$ strictly stochastically dominates $\mathbf{q}(\hat{p})$ for all $\hat{p} \in P^{*}(p)$.

The last result generalizes both Proposition 3 for the naive perception problem and Proposition 4 for the sophisticated case. When the perception error is additive as in section 4.1, the precision-maximizing perception strategy is the unbiased one, and Proposition 3 establishes that the agent is revealed to be biased upwards.

\footnotetext{
${ }^{13}$ See, for instance, Wu and Gonzalez (1996) on the elicitation of such data.
} 
Let us verify that the proposition holds for the sophisticated perception from section 4.2. We have found that, for a symmetric prior $\varphi(p)$, the payoff-maximizing perception threshold is $p^{*}<1 / 2$. The symmetry of $\varphi(p)$ implies that $p^{*}<\left(\bar{q}\left(p^{*}\right)+\underline{q}\left(p^{*}\right)\right) / 2$. For any given pair of perception levels $q<\bar{q}$, the precision-maximizing strategy partitions the probability interval to $\left[0, p^{* *}\right]$, and $\left(p^{* *}, 1\right]$, with $p^{* *}=(\bar{q}+\underline{q}) / 2$. Thus, the two perception strategies coincide for $p \notin\left(p^{*}, p^{* *}\right)$ and differ for $p \in\left(p^{*}, p^{* *}\right)$. When $p \in\left(p^{*}, p^{* *}\right)$, the payoff-maximizing agent forms perception $\bar{q}$, while the precision-maximizing agent forms $\underline{q}$. Thus, the agent is again revealed to be upward-biased.

\section{$5 \quad$ Related Perception Biases}

This section clarifies the relationship of the illusion of control to two additional perception biases.

\subsection{Optimism}

Psychologists classify the illusion of control together with optimism among so-called positive illusions (Taylor and Brown, 1988). Our model helps to clarify the relationship between the two biases. Positive belief biases are often rationalized by assuming that the agent derives felicity from holding favorable beliefs; for example, see Brunnermeier and Parker (2005), Caplin and Leahy (2001), and Köszegi (2006). In contrast, the belief distortions are purely instrumental in our model; they guide choice. This section discusses the conditions under which the instrumental bias of this paper leans in the direction of optimism.

First, note that the optimal perception strategy in any of the perception problems of section 4 is invariant to any action-independent modification of the payoffs: the solution to a perception problem under stochastic payoffs $u$ is also optimal under stochastic payoffs $\tilde{u}$, where $\tilde{u}(a, \theta)=u(a, \theta)+c_{\theta}$ for all $(a, \theta)$, and $c_{\theta}$ are action-independent constants. This is so because the optimal perception depends only on $v^{\prime \prime}(p)$ whereas the constants $c_{\theta}$ affect the value function only by an additive linear term.

When we consider the naive perception technology from section 4.1, it is natural to interpret the agent as optimistic when $v(1)>v(0)$ and when the agent's perception is biased upwards in the sense that $m^{*}(p)>p$ for all $p \in(\sigma, 1-\sigma)$. Note, however, that the optimal perception bias is caused by the ranking of control across the states, rather 
than by optimism. Adding constants to payoffs in either the high or the low state affects whether $v(1)>v(0)$ or $v(1)<v(0)$ but does not affect $v^{\prime \prime}$ and hence does not affect the optimal perception. Thus, the illusion of control does not generally entail optimism.

However, the illusion of control generates optimism under the additional assumption that the average payoff in the high state is at least as large as in the low state.

Proposition 6 (optimism). If the agent has more control in the high state than in the low one, her payoffs are normally distributed, and $\mathrm{E}_{u} u(a, h) \geq \mathrm{E}_{u} u(a, l)$, then the agent is optimistic.

Consider the worst case in which the means of the payoffs at the low and the high state are the same. The higher payoff variance in the high state implies that the optimizing agent can achieve a higher value at $h$ than at $l$. Thus, unless the payoffs in the high-control state are predictably low, the illusion of control generates optimism.

\subsection{Probability Weighting in Prospect Theory}

Two well-known stylized phenomena, overconfidence and the prospect-theory probability weighting, have opposite implications for the perception of small probabilities. Overconfidence about one's own knowledge implies an undervaluation of the small probabilities attached to unlikely outcomes, whereas prospect-theory predicts an overvaluation of small probabilities. Our model predicts both effects in two closely related settings and thus may help to clarify the respective domains of the two effects. ${ }^{14}$

We compare two settings. One of them is Example 2 and the second is the setting of Steiner and Stewart (2016). To facilitate the comparison, we describe both settings in a unified way. In both cases, the agent evaluates binary lotteries. For $a \in\{1, \ldots, n\}$, lottery $\ell_{a}=\left(r_{t}^{a}, \pi_{\theta} ; r_{d}^{a}, 1-\pi_{\theta}\right)$ pays reward $r_{\gamma}^{a}$ in outcome $\gamma \in\{t, d\}$. The rewards $r_{\gamma}^{a}$ are iid. from the standard normal density and are observed by the agent at the choice stage. The probability $\pi_{\theta}$ of the outcome $t$ is state-dependent, and $1 / 2<\pi_{l}<\pi_{h}$. (The result is the same if $\pi_{\theta}$ is below half in both states.)

1. Overprecision setting (example 2): The agent chooses one from $n$ lotteries. The lotteries differ in the rewards $r_{\gamma}^{a}$ but share the same probability parameter $\pi_{\theta}$; $u(a, \theta)=\pi_{\theta} r_{t}^{a}+\left(1-\pi_{\theta}\right) r_{d}^{a}$ for $a \in\{1, \ldots, n\}$.

\footnotetext{
${ }^{14}$ The two phenomena were discussed by the same authors within a short period of time. Kahneman and Tversky (1977) discuss overconfidence, and Kahneman and Tversky (1979) discuss the overvaluation of small probabilities.
} 


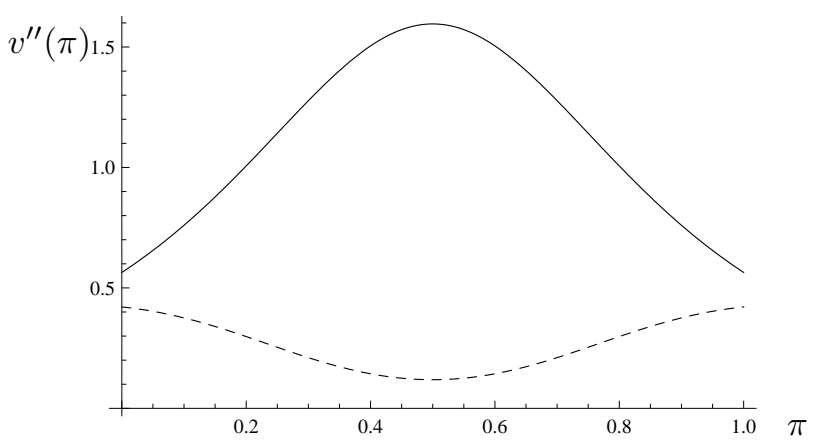

Figure 2: Solid line: $v^{\prime \prime}(\pi)$ in the overprecision setting. Dashed line: $v^{\prime \prime}(\pi)$ in the prospect-theory setting $(s=1.5)$.

2. Prospect-theory setting: The agent chooses lottery $\ell_{1}$ or a safe option that pays $s$ with certainty; $a \in\{1,2\}, u(1, \theta)=\pi_{\theta} r_{t}^{1}+\left(1-\pi_{\theta}\right) r_{d}^{1}$ and $u(2, \theta)=s$.

In both settings, the agents solve the naive perception problem (6).

In the overprecision setting, all the lotteries are bets on the same event. The uncertainty about $\pi$ thus affects payoffs to all actions. In the prospect-theory setting, the uncertainty affects only one action but not the agent's outside option.

Let $\pi(q)=\pi_{h} q+\pi_{l}(1-q)$ be the subjective probability that the agent assigns to the likely outcome $t$; note that it is above one-half. We have shown in section 4.1 that the agent's perception $q$ is biased upwards in the overprecision setting. Thus, the perceived probability of the unlikely outcome $d$ is biased downward; $\mathrm{E}[1-\pi(q) \mid p]<1-\pi(p)$. When the agent chooses from several binary bets on the same event, she underestimates the small probabilities.

The effect is reversed in the prospect-theory setting.

Proposition 7 (Steiner and Stewart, 2016). If the outside option is $s>3^{1 / 4}$, then the perception strategy that solves the perception problem (6) in the prospect-theory setting overvalues small probabilities: $\mathrm{E}[1-\pi(q) \mid p]>1-\pi(p)$.

Steiner and Stewart interpret $s$ as the maximum over the agent's alternatives to the lottery $\ell_{1}$. When the agent has sufficiently many alternatives, $s$ is much more valuable than a typical draw of the lottery, and thus $s>3^{1 / 4}$ is satisfied. The agent overvalues small probabilities that idiosyncratically affect the value of one of her actions but do not affect the alternatives.

The difference in the optimal biases across the two settings is caused by the difference 
in the pattern of the ties. Abusing notation slightly, let $v(\pi)$ be the value of the optimizing agent who correctly perceives the probability $\pi$ of the outcome $t$. Figure $2 \operatorname{depicts} v^{\prime \prime}(\pi)$ in both settings. In the overprecision setting, a tie between the first-best and the secondbest action is likely when the lottery value $r_{t}^{a} \pi+r_{d}^{a}(1-\pi)$ has a low variance, which happens when $\pi$ is close to half; $v^{\prime \prime}(\pi)$ is single-peaked with its maximum at half for the overprecision setting. In the prospect-theory setting, a tie between the lottery $\ell_{1}$ and

the high outside option $s$ is likely when the lottery value $r_{t}^{1} \pi+r_{d}^{1}(1-\pi)$ has a high variance, and hence $v^{\prime \prime}(\pi)$ is U-shaped in the prospect-theory setting. As the probability $\pi$ is bounded above half in both settings, $v^{\prime \prime}$ exhibits opposite monotonicity across the two cases. Consequently, the optimal perception strategies have the opposite biases.

Kominers et al. (2015) offer an alternative model of frictional information processing and they too derive an optimal overvaluation of small probabilities. In their example 2 , an agent allocates her unit endowment to two assets, $t$ and $d$. In our notation, the agent can choose any lottery $\left(r_{t}, \pi ; r_{d}, 1-\pi\right)$ such that $r_{t}, r_{d} \in[0,1]$ and $r_{t}+r_{d}=1$. The agent has a logarithmic utility function, and thus her expected payoff from a lottery $\left(r_{t}, \pi ; r_{d}, 1-\pi\right)$ is $\pi \log r_{t}+(1-\pi) \log r_{d}$. The optimal allocation for a given $\pi$ is $r_{t}=\pi, r_{d}=1-\pi$, and therefore the value function of the optimizing agent with correct probability perception is $v(\pi)=\pi \log \pi+(1-\pi) \log (1-\pi)$. It is easy to verify that $v^{\prime \prime}$ is U-shaped with the minimum at half. Since $v^{\prime \prime}$ has the same monotonicity properties as the value function arising in the prospect-theory setting from above, the second-best perception biases in the two settings have the same direction.

\section{General Payoff Distributions}

We assume here that the agent has more control in the high state than in the low one. For the sake of simplicity, we consider only binary action sets in this section. That is, $u(a, \theta)=u_{a}+\tau_{\theta} \eta_{a}, \tau_{h}>\tau_{l}>0, a \in\{1,2\}$. We dispense with the assumption of Gaussian payoffs and provide sufficient conditions on general payoff distributions for the monotonicity of $v^{\prime \prime}(p)$.

Let us introduce the differences $\delta=u_{2}-u_{1}$, and $\eta=\eta_{2}-\eta_{1}$; we denote their densities by $f(\delta)$ and $\phi(\eta)$. We allow for dependencies between $u_{1}$ and $u_{2}$ and between $\eta_{1}$ and $\eta_{2}$ but continue to assume that $\left(u_{1}, u_{2}\right)$ and $\left(\eta_{1}, \eta_{2}\right)$ are independent.

The next result formalizes the connection between the likelihood of the ties and the 
second derivative of the value function. A tie between the two actions arises at probability $p$ if and only if $\delta+\tau(p) \eta=0$, where $\tau(p)=\tau_{h} p+\tau_{l}(1-p)$. Thus, the likelihood of the tie at $p$ is $\int_{-\infty}^{\infty} \phi(\eta) f(-\tau(p) \eta) d \eta$. The next expression for $v^{\prime \prime}(p)$ augments the last integral by a term capturing the angles of the intersections.

\section{Lemma 4.}

$$
v^{\prime \prime}(p)=\left(\tau_{h}-\tau_{l}\right)^{2} \int_{-\infty}^{\infty} \eta^{2} \phi(\eta) f(-\tau(p) \eta) d \eta
$$

For each $\eta$, the term $\left(\tau_{h}-\tau_{l}\right)^{2} \eta^{2}=(u(2, l)-u(1, l)+u(1, h)-u(2, h))^{2}$ is the square of the angle of the intersection of the graphs of the expected payoffs $u(1, p)$ and $u(2, p)$. Thus, in line with the intuition from section $3, v^{\prime \prime}(p)$ captures the likelihood of the tie at $p$, modified for the sensitivity of the payoff difference to a variation in the belief $p$.

The last lemma allows us to establish the monotonicity of $v^{\prime \prime}(p)$ under a regularity condition without computing $v(p)$. Recall that a function is single-peaked with its maximum at $x$ if it is increasing below $x$ and decreasing above $x$.

Lemma 5. If the agent has more control in the high state than in the low one and the density $f(\delta)$ is single-peaked with its maximum at 0 , then $v^{\prime \prime}(p)$ is decreasing on $(0,1)$.

The difference $\delta=u_{2}-u_{1}$ has a single-peaked density when $u_{1}$ and $u_{2}$ are drawn iid. from many common densities, including the normal or Pareto densities. Additionally, we provide a condition on the joint distribution of $u_{1}$ and $u_{2}$ that is sufficient for the single-peakedness of $f(\delta)$. Recall that a function $h: \mathbf{R}^{2} \longrightarrow \mathbf{R}$ is supermodular if for any $x, y, x^{\prime}, y^{\prime} \in \mathbf{R}^{4}$ :

$$
h\left(\max \left\{x, x^{\prime}\right\}, \max \left\{y, y^{\prime}\right\}\right)+h\left(\min \left\{x, x^{\prime}\right\}, \min \left\{y, y^{\prime}\right\}\right) \geq h(x, y)+h\left(x^{\prime}, y^{\prime}\right) .
$$

Let $g\left(u_{1}, u_{2}\right)$ denote the joint density of $u_{1}$ and $u_{2}$. We say that $g$ is symmetric if $g\left(u_{1}, u_{2}\right)=g\left(u_{2}, u_{1}\right)$ for every $\left(u_{1}, u_{2}\right)$.

Lemma 6. If $g$ is symmetric and supermodular, then $f(\delta)$ is single-peaked with maximum at 0 .

The supermodularity assumption is related to the definition of affiliated random variables (Milgrom and Weber, 1982). The assumption generalizes the notion of positive correlation between $u_{1}$ and $u_{2}$ and corresponds to situations that may arise when the two 
payoff terms $u_{1}$ and $u_{2}$ are generated by a common process. ${ }^{15}$

The following result concludes this section by summarizing conditions under which we obtain overconfidence.

Corollary 4. If the agent has more control in the high state than in the low one and the joint density $g$ is both symmetric and supermodular, then $v^{\prime \prime}(p)$ is decreasing and the solution of the naive perception problem (6) is biased upwards: $m^{*}(p)>p$ and $\mathrm{E}[q \mid p]>p$ for all $p \in(\sigma, 1-\sigma)$. If, additionally, $\varphi(p)$ is symmetric around $1 / 2$, then the threshold probability $p^{*}$ that solves the sophisticated perception problem (8) is less than $1 / 2$.

\section{Discussion}

The designer of the decision process and the agent from our model can be interpreted in different ways. The designer may stand for the biological or cultural evolution of information processing whereas the agent may represent the individual. Alternatively, the model may be applied to the problem of the design and organization of a firm subject to frictions in its internal communication.

The model may help to inform us of the relevant domains of common behavioral phenomena. Though there is considerable consensus on both overconfidence and prospecttheory probability weighting, these two phenomena make conflicting predictions for the perception of small probabilities. A joint microfoundation may help to delineate this aspect of the two phenomena.

Models that provide microfoundations for behavioral biases can contribute to normative discussions of debiasing. Our model suggests that a bias relative to the precisionmaximizing perception may be optimal and thus does not justify an intervention on its own. On the other hand, the model may inform us of circumstances under which an intervention into the decision process may be beneficial. A standard evolutionary argument for interventions applies: the modern agent's environment may differ from the one she is adapted to. In our case, we have assumed that the utility function $u$ and the probability $p$ are independent random variables. This is reasonable if the decision problem is generated by a nonstrategic process, but the independence assumption is violated in a market environment in which the reward for and probability of an outcome of a financial bet are typically negatively correlated.

\footnotetext{
${ }^{15}$ Random variables are affiliated if the logarithm of their joint density is supermodular. Our assumption applies to the density instead of the log-density.
} 
The model suggests that an intervention may be justified even if the agent's environment has not changed since adaptation took place. To see this, consider a stylized example in which the intervening outsider knows more about the agent's decision problem than the perception designer has known. Assuming that the choices made by a healthy individual have a larger impact on her well-being than the choices made by a sick individual in most of the decision problems, the model predicts that health perception will be biased toward the denial of sickness. The assumption, however, may fail for a finer classification of the diseases. If some of the diseases are treatable by an appropriate action whereas some are not treatable, then the individual suffering from a treatable disease has considerable control over her well-being; the second-best perception should exhibit some hypochondria in the domain of treatable diseases and denial in the domain of nontreatable diseases. If the perception of one's own health status has been designed under a coarse classification of the diseases, whereas the intervening outsider distinguishes more finely between treatable and nontreatable diseases, then the outsider may call for an intervention even if the distribution of the diseases has not changed. We hope to return to a systematic study of the normative implications of the model in a future work.

\section{A Proofs}

Proof of Proposition 1 is below the proof of Lemma 2.

Proof of Lemma 2. Let $u_{a} \sim \mathcal{N}\left(\mu, \omega^{2}\right)$ and $\eta_{a} \sim \mathcal{N}\left(\nu, \omega^{\prime 2}\right)$. Then

$$
u(a, p) \sim \mathcal{N}\left(\mu+\tau(p) \nu, \omega^{2}+\tau^{2}(p) \omega^{\prime 2}\right)
$$

where $\tau(p)=(1-p) \tau_{l}+p \tau_{h}$. Thus, $v(p)=\mu+\tau(p) \nu+c \sigma(p)$ where $\sigma(p)=\sqrt{\omega^{2}+\tau^{2}(p) \omega^{\prime 2}}$ is the standard deviation of $u(a, p)$. Observing that the linear term does not affect $v^{\prime \prime}(p)$, we have that $v^{\prime \prime}(p)=c \sigma^{\prime \prime}(p)$. Using the equality $\sigma(p)=\sqrt{c_{1}+c_{2} p+c_{3} p^{2}}$ for some constants $c_{1}, c_{2}$, and $c_{3}$, we obtain that

$$
v^{\prime \prime}(p)=c \frac{d^{2}}{d p^{2}} \sqrt{c_{1}+c_{2} p+c_{3} p^{2}}=c \frac{-c_{2}^{2}+4 c_{1} c_{3}}{4} \sigma^{-3}(p) .
$$

Since the value function is the expectation over the upper envelopes of linear functions and thus convex and since $\sigma(p)$ is positive, it must be the case that $c \frac{-c_{2}^{2}+4 c_{1} c_{3}}{4}$ is positive. By the assumption that $\tau_{h}>\tau_{l}$, the function $\tau(p)$ and hence also $\sigma(p)$ are increasing. 
Thus, $v^{\prime \prime}(p)$ is decreasing in $p$.

Proof of Proposition 1. Example 1: Identification of $-c_{a}$ with $u_{a}$ and $b_{a}$ with $\eta_{a}$, respectively, from (1) implies that the agent has more control in the high state than in the low one.

Example 2: Let $u_{a}=\left(r_{t}^{a}+r_{d}^{a}\right) / 2$, and let $\eta_{a}=r_{t}^{a}-r_{d}^{a}$; notice that they are independent. Then, $u(a, \theta)=u_{a}+\left(\pi_{\theta}-1 / 2\right) \eta_{a}$. The assumption that $\pi_{h}>\pi_{l}>1 / 2$ implies that the agent has more control in the high state than in the low one.

Example 3: Assume that the term $e_{a}$ is drawn from $\mathcal{N}\left(\mu, \omega^{2}\right)$. Note that $u(a, \theta) \sim$ $\mathcal{N}\left(\mu+y, \omega^{2}+\frac{\gamma_{\theta}^{2}\left(1+1 / \gamma_{\theta}\right)}{\left(1+\gamma_{\theta}\right)^{2}}\right)=\mathcal{N}\left(\mu+y, \omega^{2}+\frac{\gamma_{\theta}}{\gamma_{\theta}+1}\right)$. Thus $u(a, \theta)$ equals in distribution $u_{a}+\sqrt{\frac{\gamma_{\theta}}{\gamma_{\theta}+1}} \eta_{a}$, where $u_{a} \sim \mathcal{N}\left(\mu+y, \omega^{2}\right), \eta_{a} \sim \mathcal{N}(0,1)$, and $u_{a}, \eta_{a}$ are independent. By the assumed monotonicity we have $\gamma_{h}>\gamma_{l}$, it follows that $\sqrt{\frac{\gamma_{h}}{\gamma_{h}+1}}>\sqrt{\frac{\gamma_{l}}{\gamma_{l}+1}}$, and the agent has more control in the high state than in the low one.

The result now follows for all three examples from Corollary 1.

Proof of Proposition 2. By Lemma 1,

$$
\mathrm{E}_{\varepsilon} L(p, m+\varepsilon)=\mathrm{E}_{\varepsilon}\left[\int_{p}^{m+\varepsilon} v^{\prime \prime}(s)(s-p) d s\right] .
$$

Since

$$
\frac{d}{d m} \mathrm{E}_{\varepsilon}\left[\int_{p}^{m+\varepsilon} v^{\prime \prime}(s)(s-p) d s\right]=\mathrm{E}_{\varepsilon}\left[v^{\prime \prime}(m+\varepsilon)(m+\varepsilon-p)\right],
$$

the first-order condition for the interior optimal $m$ gives (7).

Proof of Proposition 3. By Lemma 1,

$$
\frac{d}{d m} \mathrm{E}_{\varepsilon} L(p, m+\varepsilon)=\mathrm{E}_{\varepsilon}\left[v^{\prime \prime}(m+\varepsilon)(m+\varepsilon-p)\right] .
$$

Assume by contradiction that $m \leq p$. Since $v^{\prime \prime}(p)$ is decreasing and $m-\sigma-p<0$, we have:

$$
\frac{d}{d m} \mathrm{E}_{\varepsilon} L(p, m+\varepsilon)<\mathrm{E}_{\varepsilon}\left[v^{\prime \prime}(m+\sigma)(m+\varepsilon-p)\right]=v^{\prime \prime}(m+\sigma)(m-p) \leq 0 .
$$

Since the constraint $m \leq 1-\sigma$ is not binding, the established inequality, $\frac{d}{d m} \mathrm{E}_{\varepsilon} L(p, m+$ $\varepsilon)<0$, violates the necessary first-order condition for the loss minimization.

Proof of Lemma 3. For any given $m(\cdot)$, the payoff optimization implies that $c(u, m)=$ 
$a_{q(m), u}^{*}$, where $q(m)=\mathrm{E}[p \mid m(p)=m]$. For any two posteriors $\underline{q}<\bar{q}$, the difference between the losses across the two posteriors is

$$
L\left(p, q^{\prime}\right)-L(p, q)=\int_{q}^{q^{\prime}} v^{\prime \prime}(s)(s-p) d s .
$$

The last expression is linear in $p$, and therefore there exists $p^{*} \in[0,1]$ such that the posterior $q$ is optimal below $p^{*}$ and $\bar{q}$ is optimal above $p^{*}$.

Proof of Proposition 4. We prove the statement of the proposition under the condition

$$
v^{\prime \prime}(p)>v^{\prime \prime}(1-p) \text { for all } p<1 / 2 \text {. }
$$

The monotonicity of $v^{\prime \prime}(p)$ assumed in the proposition implies (11).

Recall that $\underline{q}\left(p^{*}\right)=\mathrm{E}\left[p \mid p<p^{*}\right]$ and $\bar{q}\left(p^{*}\right)=\mathrm{E}\left[p \mid p \geq p^{*}\right]$. Note that the symmetry of the density of $p$ implies $q\left(p^{*}\right)<1 / 2<\bar{q}\left(p^{*}\right)$ for any $p^{*} \in(0,1)$. Define

$$
q\left(p ; p^{*}\right)= \begin{cases}\bar{q}\left(p^{*}\right) & \text { if } p \geq p^{*}, \\ \underline{q}\left(p^{*}\right) & \text { if } p<p^{*} .\end{cases}
$$

$q\left(p, p^{*}\right)$ denotes the agent's posterior when the objective probability is $p$ and the threshold probability is $p^{*}$.

We compare the payoff of the agent who uses the threshold $p^{*}$ with the payoff of an agent who has only the prior information about $p$. By the symmetry of the prior density of $p$, this last agent attaches probability $\mathrm{E} p=1 / 2$ to the high state and her expected payoff is:

$$
v(1 / 2)=\mathrm{E}_{p} V\left(q\left(p ; p^{*}\right), 1 / 2\right) .
$$

The expected payoff to the agent who observes whether $p<p^{*}$ or $p \geq p^{*}$ is $\mathrm{E}_{p} v\left(q\left(p ; p^{*}\right)\right)$ and the optimal partition maximizes this value over $p^{*}$. We rewrite this objective function as:

$$
\begin{aligned}
\mathrm{E}_{p} v\left(q\left(p ; p^{*}\right)\right) & =v(1 / 2)+\mathrm{E}_{p}\left[v\left(q\left(p ; p^{*}\right)\right)-v(1 / 2)\right] \\
& =v(1 / 2)+\mathrm{E}_{p}\left[v\left(q\left(p ; p^{*}\right)\right)-V\left(q\left(p ; p^{*}\right), 1 / 2\right)\right] \\
& =v(1 / 2)+\mathrm{E}_{p} L\left(q\left(p ; p^{*}\right), 1 / 2\right) .
\end{aligned}
$$




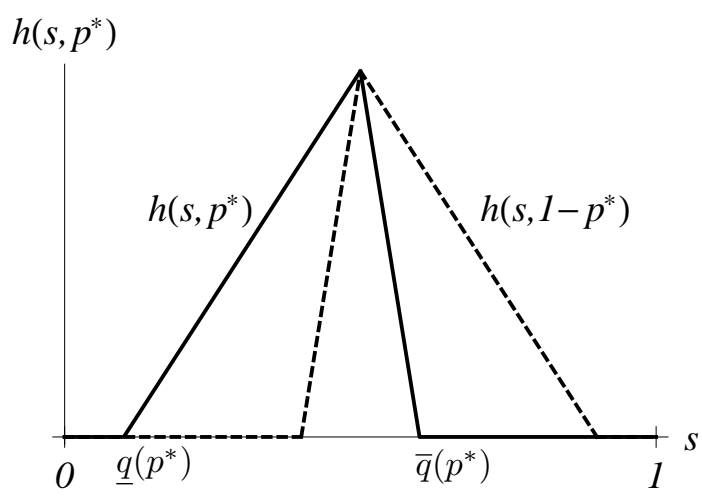

Figure 3: $p^{*}<1 / 2$. Thick line: $h\left(s, p^{*}\right)$. Dashed line: $h\left(s, 1-p^{*}\right)=h\left(1-s, p^{*}\right)$. Note that $h\left(s, 1-p^{*}\right) \leq h\left(s, p^{*}\right)$ for $s \leq 1 / 2$.

Notice that $\operatorname{Pr}\left(p<p^{*}\right)=\frac{\bar{q}\left(p^{*}\right)-1 / 2}{\bar{q}\left(p^{*}\right)-\underline{q}\left(p^{*}\right)}$ and $\operatorname{Pr}\left(p \geq p^{*}\right)=\frac{1 / 2-q\left(p^{*}\right)}{\bar{q}\left(p^{*}\right)-\underline{q}\left(p^{*}\right)}$. Thus, the threshold $p^{*}$ maximizes the objective

$$
o\left(p^{*}\right):=\frac{\bar{q}\left(p^{*}\right)-1 / 2}{\bar{q}\left(p^{*}\right)-\underline{q}\left(p^{*}\right)} L\left(\underline{q}\left(p^{*}\right), 1 / 2\right)+\frac{1 / 2-\underline{q}\left(p^{*}\right)}{\bar{q}\left(p^{*}\right)-\underline{q}\left(p^{*}\right)} L\left(\bar{q}\left(p^{*}\right), 1 / 2\right) .
$$

Substituting (2) for $L(q, 1 / 2)$ into (12) gives

$$
o\left(p^{*}\right)=\int_{0}^{1} v^{\prime \prime}(s) h\left(s, p^{*}\right) d s
$$

where

$$
h\left(s, p^{*}\right)= \begin{cases}0 & \text { if } p<\underline{q}\left(p^{*}\right), \\ \frac{\bar{q}\left(p^{*}\right)-1 / 2}{\bar{q}\left(p^{*}\right)-\underline{q}\left(p^{*}\right)}\left(s-\underline{q}\left(p^{*}\right)\right) & \text { if } \underline{q}\left(p^{*}\right) \leq p<1 / 2, \\ \frac{1 / 2-\underline{q}\left(p^{*}\right)}{\bar{q}\left(p^{*}\right)-\underline{q}\left(p^{*}\right)}\left(\bar{q}\left(p^{*}\right)-s\right) & \text { if } 1 / 2 \leq p<\bar{q}\left(p^{*}\right), \\ 0 & \text { if } p>\bar{q}\left(p^{*}\right) .\end{cases}
$$

The function $h\left(s, p^{*}\right)$ is continuous piecewise-linear in $s$, as depicted in figure 3 .

Let us now prove that $o\left(p^{*}\right)>o\left(1-p^{*}\right)$ when $p^{*}<1 / 2$. For such $p^{*}$, the symmetry of the density of $p$ implies that $\underline{q}\left(p^{*}\right)<1-\bar{q}\left(p^{*}\right)$. The definition (13) of $h$ then implies

$$
h\left(s, p^{*}\right)>h\left(1-s, p^{*}\right) \text { for all } p^{*}<1 / 2, s<1 / 2 .
$$

Note also that by the symmetry of $\varphi(p)$, it follows that:

$$
h\left(s, p^{*}\right)=h\left(1-s, 1-p^{*}\right) \text { for all } p^{*}, s \in(0,1) .
$$


We now obtain

$$
\begin{aligned}
o\left(p^{*}\right)-o\left(1-p^{*}\right) & =\int_{0}^{1} v^{\prime \prime}(s)\left(h\left(s, p^{*}\right)-h\left(s, 1-p^{*}\right)\right) d s \\
& =\int_{0}^{1} v^{\prime \prime}(s)\left(h\left(s, p^{*}\right)-h\left(1-s, p^{*}\right)\right) d s \\
& =\int_{0}^{1 / 2}\left(v^{\prime \prime}(s)-v^{\prime \prime}(1-s)\right)\left(h\left(s, p^{*}\right)-h\left(1-s, p^{*}\right)\right) d s
\end{aligned}
$$

The first bracket in the last integral is positive by condition (11). When $p^{*}<1 / 2$, the second bracket is positive by (14). We have thus established that $p^{*}>1 / 2$ cannot be optimal. Hence, $p^{*} \leq 1 / 2$.

It remains for us to prove that $p^{*} \neq 1 / 2$. Let

$$
w\left(p^{*}, q_{l}, q_{h}\right)=\int_{0}^{p^{*}} V\left(p, q_{l}\right) \varphi(p) d p+\int_{p^{*}}^{1} V\left(p, q_{h}\right) \varphi(p) d p
$$

stand for the expected payoff when the agent forms belief $q_{l}$ for $p<p^{*}$ and belief $q_{h}$ for $p \geq p^{*}$. The agent's objective is $\mathrm{E}_{p} v\left(q\left(p ; p^{*}\right)\right)=w\left(p^{*}, \underline{q}\left(p^{*}\right), \bar{q}\left(p^{*}\right)\right)$. Notice that

$$
\begin{aligned}
\frac{d}{d p^{*}} \mathrm{E}_{p} v\left(q\left(p ; p^{*}\right)\right) & =w_{1}\left(p^{*}, \underline{q}\left(p^{*}\right), \bar{q}\left(p^{*}\right)\right) \\
& =\varphi\left(p^{*}\right)\left(V\left(p^{*}, \underline{q}\left(p^{*}\right)\right)-V\left(p^{*}, \bar{q}\left(p^{*}\right)\right)\right) \\
& =\varphi\left(p^{*}\right)\left(V\left(p^{*}, \underline{q}\left(p^{*}\right)\right)-v\left(p^{*}\right)-V\left(p^{*}, \bar{q}\left(p^{*}\right)\right)+v\left(p^{*}\right)\right) \\
& =\varphi\left(p^{*}\right)\left(L\left(p^{*}, \bar{q}\left(p^{*}\right)\right)-L\left(p^{*}, \underline{q}\left(p^{*}\right)\right)\right),
\end{aligned}
$$

where $w_{1}$ in the first line stands for the partial derivative with respect to the first argument. The first equality follows from the optimality of the Bayesian posteriors and from the Envelope Theorem. Observe that, by the symmetry of $\varphi$, it follows that $\underline{q}(1 / 2)=1-$ $\bar{q}(1 / 2)$. Thus,

$$
\begin{aligned}
\left.\frac{d}{d p^{*}} \mathrm{E}_{p} v\left(q\left(p ; p^{*}\right)\right)\right|_{p^{*}=1 / 2} & =\varphi(1 / 2)(L(1 / 2,1-\underline{q}(1 / 2))-L(1 / 2, \underline{q}(1 / 2))) \\
& =\varphi(1 / 2)\left(\int_{1 / 2}^{1-\underline{q}(1 / 2)} v^{\prime \prime}(s)(s-1 / 2) d s-\int_{1 / 2}^{\underline{q}(1 / 2)} v^{\prime \prime}(s)(s-1 / 2) d s\right) \\
& =\varphi(1 / 2)\left(\int_{1 / 2}^{1-\underline{q}(1 / 2)}\left(v^{\prime \prime}(s)-v^{\prime \prime}(1-s)\right)(s-1 / 2) d s\right),
\end{aligned}
$$

and the last integral is negative by condition (11). Thus, $p^{*}=1 / 2$ cannot satisfy the 
first-order condition necessary for the optimum.

Proof of Proposition 5. We compare the solutions to two problems. Let $Q^{*}=\{\mathbf{q}(p): p \in$ $[0,1]\}$ be the set of the stochastic perceptions used by the agent. The payoff-maximizing perception must solve

$$
\mathbf{q}(p) \in \arg \max _{\mathbf{q} \in Q^{*}} \mathrm{E}[-L(p, \mathbf{q})]
$$

In contrast, the precision-maximizing perception solves

$$
\hat{\mathbf{q}}(p) \in \arg \max _{\mathbf{q} \in Q^{*}} \mathrm{E}[-\hat{L}(p, \mathbf{q})]
$$

where

$$
\hat{L}(p, q)=\frac{v^{\prime \prime}(p)}{2}(q-p)^{2}=\int_{p}^{q} v^{\prime \prime}(p)(s-p) d s
$$

The expression $\hat{L}(p, q)$ differs from the square of the perception error only by the multiplicative factor $v^{\prime \prime}(p) / 2$, which does not affect the solution of the precision optimization.

First, note that the objective function $-L(p, q)$ has increasing differences in $(p, q)$. Indeed, by Lemma 1,

$$
-L\left(p, q^{\prime}\right)+L(p, q)=\int_{q}^{q^{\prime}}(p-s) v^{\prime \prime}(s) d s
$$

which increases in $p$ when $q^{\prime}>q$. Thus, the objective $\mathrm{E}[-L(p, \mathbf{q})]$ has increasing differences (with $\mathbf{q}$ ordered by the stochastic dominance order). Therefore, by Theorem 1 in Van Zandt (2002), the payoff-maximizing perception $\mathbf{q}(p)$ is non decreasing in $p$ with respect to the stochastic order.

Further, note that the objective $-L(p, q)$ has larger payoff differences than the objective $-\hat{L}(p, q)$. Indeed, using Lemma 1 ,

$$
-L\left(p, q^{\prime}\right)+L(p, q)-\left(-\hat{L}\left(p, q^{\prime}\right)+\hat{L}(p, q)\right)=\int_{q}^{q^{\prime}}(s-p)\left(v^{\prime \prime}(p)-v^{\prime \prime}(s)\right) d s .
$$

The last expression is positive if $q^{\prime}>q$ because $v^{\prime \prime}$ is decreasing. Thus, the objective $\mathrm{E}[-L(p, \mathbf{q})]$ has larger payoff differences than $\mathrm{E}[-\hat{L}(p, \mathbf{q})]$. This means that, using $\succ$ to denote the first-order stochastic dominance order, for any $p$ and $\mathbf{q}^{\prime} \succ \mathbf{q}$,

$$
\mathrm{E}\left[-L\left(p, \mathbf{q}^{\prime}\right)\right]-\mathrm{E}[-L(p, \mathbf{q})]>\mathrm{E}\left[-\hat{L}\left(p, \mathbf{q}^{\prime}\right)\right]-\mathrm{E}[-\hat{L}(p, \mathbf{q})]
$$


Therefore, by Theorem 1 in Van Zandt (2002), $\mathbf{q}(p) \succeq \hat{\mathbf{q}}(p)$ for all $p$ and for all solutions $\mathbf{q}(p)$ and $\hat{\mathbf{q}}(p)$ of the two problems. Since, by assumption, $\mathbf{q}(p)$ does not solve the precision optimization problem, it must be that $\mathbf{q}(p) \succ \hat{\mathbf{q}}(p)$. Since $\hat{\mathbf{q}}(p) \in Q^{*}$, there exists $\hat{p} \in[0,1]$ such that $\hat{\mathbf{q}}(p)=\mathbf{q}(\hat{p})$ and because $\mathbf{q}(p)$ is non decreasing, $\hat{p}<p$ for all such $\hat{p}$.

Proof of Proposition 6. It suffices to prove the proposition for the case $\mathrm{E}_{u}[u(a, h)]=$ $\mathrm{E}_{u}[u(a, l)]$, because $v(1)$ increases with an upward shift of the distribution of $u(a, h)$. More control in the high state implies that $\operatorname{Var}_{u}[u(a, h)]>\operatorname{Var}_{u}[u(a, l)]$. Together with $\mathrm{E}_{u}[u(a, h)]=\mathrm{E}_{u}[u(a, l)]$ and normality, this implies that the payoffs in the high state are a mean-preserving spread of the payoffs in the low state. By convexity of the max function,

$$
v(1)=\mathrm{E} \max _{a}\{u(a, h)\}>\operatorname{E} \max _{a}\{u(a, l)\}=v(0)
$$

By Corollary 2, the optimal perception is biased upwards. Hence, the bias is optimistic.

Proposition 7 was proved in Steiner and Stewart (2016); see their Theorem 1. Here, we briefly sketch the proof of the proposition using the analytical approach of this paper. Let $v(p)=\mathrm{E}_{r_{t}, r_{d}} \max \left\{\pi(p) r_{t}+(1-\pi(p)) r_{d}, s\right\}$. By Proposition 3 (reversing the labels of the states), it suffices to prove that $v^{\prime \prime}(p)$ is increasing. Since $\pi(p)$ is a linear mapping that attains values in $(1 / 2,1)$, it suffices to prove that $\tilde{v}^{\prime \prime}(\pi)$ is increasing on $(1 / 2,1)$, where $\tilde{v}(\pi)=\mathrm{E}_{r_{t}, r_{d}} \max \left\{\pi r_{t}+(1-\pi) r_{d}, s\right\}$. Observe that

$$
\tilde{v}^{\prime}(\pi)=\mathrm{E}_{r_{t}, r_{d}}\left[\left(r_{t}-r_{d}\right) 1_{\pi r_{t}+(1-\pi) r_{d} \geq s}\right]
$$

where $1_{\pi r_{t}+(1-\pi) r_{d} \geq s}$ is 1 if $\pi r_{t}+(1-\pi) r_{d} \geq s$, and zero otherwise. One can verify that $\tilde{v}^{\prime \prime}(\pi)=\lambda(\pi) w(\pi)$, where $w(\pi)$ is the likelihood of the tie $\pi r_{t}+(1-\pi) r_{d}=s$, and $\lambda(\pi)=\mathrm{E}\left[\left(r_{t}-r_{d}\right)^{2} \mid \pi r_{t}+(1-\pi) r_{d}=s\right]$. Steiner and Stewart (2016) provide explicit forms of $w(\pi)$ and $\lambda(\pi)$ and prove that if $s>3^{1 / 4}$ then $\lambda(\pi) w(\pi)$ is increasing on $(1 / 2,1)$; see their Lemma 2.

Proof of Lemma 4. Using insight (3) from the beginning of the proof of Lemma 1, it follows 
that:

$$
\begin{aligned}
v^{\prime}(p) & =\mathrm{E}_{u}\left[u\left(a_{p, u}^{*}, h\right)-u\left(a_{p, u}^{*}, l\right)\right] \\
& =\mathrm{E}_{u}\left[u_{a_{p, u}^{*}}+\tau_{h} \eta_{a_{p, u}^{*}}-u_{a_{p, u}^{*}}-\tau_{l} \eta_{a_{p, u}^{*}}\right] \\
& =\left(\tau_{h}-\tau_{l}\right) \mathrm{E}_{u} \eta_{a_{p, u}^{*}} .
\end{aligned}
$$

Recall that $\tau(p)=p \tau_{h}+(1-p) \tau_{l}$. The agent chooses action 1 if $-\tau(p) \eta>\delta$, and chooses action 2 if $-\tau(p) \eta<\delta$. Thus,

$$
\begin{aligned}
\mathrm{E}_{u} \eta_{a_{p, u}^{*}} & =\int_{\left\{\left(\eta_{1}, \eta_{2}, \delta\right):-\tau(p) \eta>\delta\right\}} \eta_{1} f(\delta) \phi(\eta) d \delta d \eta_{1} d \eta_{2}+\int_{\left\{\left(\eta_{1}, \eta_{2}, \delta\right):-\tau(p) \eta<\delta\right\}} \eta_{2} f(\delta) \phi(\eta) d \delta d \eta_{1} d \eta_{2} \\
& =\int_{\mathbf{R}^{2}} \phi(\eta)\left(\int_{-\infty}^{-\tau(p) \eta} \eta_{1} f(\delta) d \delta+\int_{-\tau(p) \eta}^{\infty} \eta_{2} f(\delta) d \delta\right) d \eta_{1} d \eta_{2},
\end{aligned}
$$

and

$$
\begin{aligned}
\frac{d}{d p} \mathrm{E}_{u} \eta_{a_{p, u}^{*}} & =\int_{\mathbf{R}^{2}} \phi(\eta)\left(-\eta_{1} f(-\tau(p) \eta)\left(\tau_{h}-\tau_{l}\right) \eta+\eta_{2} f(-\tau(p) \eta)\left(\tau_{h}-\tau_{l}\right) \eta\right) d \eta_{1} d \eta_{2} \\
& =\left(\tau_{h}-\tau_{l}\right) \int_{-\infty}^{\infty} \phi(\eta) \eta^{2} f(-\tau(p) \eta) d \eta
\end{aligned}
$$

Substitution of the last line into (15) gives (10).

Proof of Lemma 5. The result follows from (10): it follows from $\tau_{h}>\tau_{l}$ that $\tau(p)$ is increasing in $p$, which implies that $f(-\tau(p) \eta)$ is decreasing in $p$ for all $p$ and $\eta$.

Proof of Lemma 6. The symmetry of $g$ implies $f(\delta)=f(-\delta)$ for all $\delta$. Thus, for arbitrary $t \in \mathbf{R}$

$$
f(\delta)=\int_{-\infty}^{\infty} g(x+t, x+t+|\delta|) d x=\int_{-\infty}^{\infty} g(x+t, x+t-|\delta|) d x .
$$

Consider $\delta, \delta^{\prime}$ such that $\left|\delta^{\prime}\right|>|\delta|$, and let $z=\left|\delta^{\prime}\right|-|\delta|>0$. Using (16), we get:

$2\left(f(\delta)-f\left(\delta^{\prime}\right)\right)=\int_{-\infty}^{\infty} g(u, u+|\delta|)+g(u-z, u-z-|\delta|)-g\left(u, u-\left|\delta^{\prime}\right|\right)-g\left(u-z, u-z+\left|\delta^{\prime}\right|\right) d u$.

Since

$$
u+|\delta|=u-z+\left|\delta^{\prime}\right| \text { and } u-\left|\delta^{\prime}\right|=u-z-|\delta|,
$$

the supermodularity of $g$ implies that the last integrand is positive. 


\section{References}

Bénabou, R. and J. Tirole (2002). Self-confidence and personal motivation. Quarterly Journal of Economics, 871-915.

Brunnermeier, M. K. and J. A. Parker (2005). Optimal expectations. American Economic Review 95(3), 1092-1118.

Caplin, A. and J. Leahy (2001). Psychological expected utility theory and anticipatory feelings. Quarterly Journal of Economics, 55-79.

Compte, O. and A. Postlewaite (2004). Confidence-enhanced performance. American Economic Review, 1536-1557.

Compte, O. and A. Postlewaite (2012). Belief formation. PIER Working Paper 12-027, University of Pennsylvania.

Cover, T. M. and J. A. Thomas (2006). Elements of Information Theory (second ed.). Wiley Series in Telecomunications. New York: Wiley.

Dow, J. (1991). Search decisions with limited memory. The Review of Economic Studies $58(1), 1-14$.

Haselton, M. G. and D. M. Buss (2000). Error management theory: A new perspective on biases in cross-sex mind reading. Journal of Personality and Social Psychology 78(1), $81-91$.

Herold, F. and N. Netzer (2010). Probability weighting as evolutionary second-best. University of Zurich Socioeconomic Institute Working Paper 1005.

Hirshleifer, D. (2001). Investor psychology and asset pricing. The Journal of Finance 56(4), 1533-1597.

Johnson, D. D., D. T. Blumstein, J. H. Fowler, and M. G. Haselton (2013). The evolution of error: Error management, cognitive constraints, and adaptive decision-making biases. Trends in Ecology 83 Evolution 28(8), 474-481.

Johnson, D. D. and J. H. Fowler (2011). The evolution of overconfidence. Nature $477(7364), 317-320$. 
Kahneman, D. and A. Tversky (1977). Intuitive prediction: Biases and corrective procedures. Technical report, DTIC Document.

Kahneman, D. and A. Tversky (1979). Prospect theory: An analysis of decision under risk. Econometrica 47(2), 263-291.

Kominers, S., X. Mu, and A. Peysakhovich (2015). Paying (for) attention: The impact of information processing costs on bayesian inference. Technical report, Harvard University.

Köszegi, B. (2006). Ego utility, overconfidence, and task choice. Journal of the European Economic Association 4(4), 673-707.

Langer, E. J. (1975). The illusion of control. Journal of Personality and Social Psychology 32(2), 311.

Lipman, B. L. (1995). Information processing and bounded rationality: A survey. Canadian Journal of Economics, 42-67.

Menezes, C., C. Geiss, and J. Tressler (1980). Increasing downside risk. American Economic Review 70(5), 921-932.

Milgrom, P. R. and R. J. Weber (1982). A theory of auctions and competitive bidding. Econometrica, 1089-1122.

Moore, D. A. and P. J. Healy (2008). The trouble with overconfidence. Psychological Review 115(2), 502-517.

Mullainathan, S. (2002). A memory-based model of bounded rationality. Quarterly Journal of Economics, 735-774.

Nettle, D. (2004). Adaptive illusions: optimism, control and human rationality. In D. Evans and P. Cruse (Eds.), Emotion, Evolution and Rationality, pp. 193-208. Oxford University Press.

Netzer, N. (2009). Evolution of time preferences and attitudes toward risk. American Economic Review 99(3), 937-955.

Ortoleva, P. and E. Snowberg (2015). Overconfidence in political behavior. American Economic Review 105(2), 504-535. 
Puri, M. and D. T. Robinson (2007). Optimism and economic choice. Journal of Financial Economics 86(1), 71-99.

Rayo, L. and G. S. Becker (2007). Evolutionary efficiency and happiness. Journal of Political Economy 115(2), 302-337.

Robson, A. J. (2001). Why would nature give individuals utility functions? Journal of Political Economy 109(4), 900-914.

Robson, A. J. and L. Samuelson (2010). The evolutionary foundations of preferences. In Handbook of social economics, Volume 1, pp. 221-310. Elsevier, Amsterdam: North Holland.

Shannon, C. (1959). Coding theorems for a discrete source with a fidelity criterion. Institute of Radio Engineers National Convention Record part 4 7(142-163), 142-163.

Shannon, C. E. (1948). A mathematical theory of communication. The Bell System Technical Journal 27, 3-55.

Simon, H. A. (1955). A behavioral model of rational choice. Quarterly Journal of Economics, 99-118.

Sims, C. A. (1998). Stickiness. In Carnegie-Rochester Conference Series on Public Policy, Volume 49, pp. 317-356. Elsevier.

Sims, C. A. (2003). Implications of rational inattention. Journal of Monetary Economics 50(3), 665-690.

Steiner, J. and C. Stewart (2016). Perceiving prospects properly. American Economic Review 106(7), 1601-31.

Taylor, S. E. and J. D. Brown (1988). Illusion and well-being: a social psychological perspective on mental health. Psychological Bulletin 103(2), 193.

Van Zandt, T. (2002). An introduction to monotone comparative statics. Insead, Fontainebleau.

Wang, Z., A. C. Bovik, H. R. Sheikh, and E. P. Simoncelli (2004). Image quality assessment: from error visibility to structural similarity. IEEE Transactions on Image Processing 13(4), 600-612. 
Wilson, A. (2014). Bounded memory and biases in information processing. Econometrica 82(6), 2257-2294.

Woodford, M. (2012a). Inattentive valuation and reference-dependent choice. Columbia University, New York.

Woodford, M. (2012b). Prospect theory as efficient perceptual distortion. American Economic Review 102(3), 41-46.

Wu, G. and R. Gonzalez (1996). Curvature of the probability weighting function. Management Science 42(12), 1676-1690. 


\begin{abstract}
Abstrakt
Studujeme systematické chyby ve vnímání v rámci suboptimálních strategií vnímání. Ekonomický agent správně pozoruje parametr, který je relevantní pro mnoho jeho rozhodnutí, ale není schopen zapamatovat si veškeré informace do doby rozhodnutí. Konstruktér rozhodovacího procesu vybírá strategii vnímání, která determinuje chyby ve vnímání. Pakliže je ztráta informací nevyhnutelná z titulu omezeného vnímání, pak (při splnění dalších podmínek) optimální strategie vnímání obsahuje iluzi kontroly, prŕlišnou sebedůvěru a optimismus.
\end{abstract}




\section{Working Paper Series}

ISSN 1211-3298

Registration No. (Ministry of Culture): E 19443

Individual researchers, as well as the on-line and printed versions of the CERGE-EI Working Papers (including their dissemination) were supported from institutional support RVO 67985998 from Economics Institute of the ASCR, v. v. i.

Specific research support and/or other grants the researchers/publications benefited from are acknowledged at the beginning of the Paper.

(c) Olivier Gossner and Jakub Steiner, 2016

All rights reserved. No part of this publication may be reproduced, stored in a retrieval system or transmitted in any form or by any means, electronic, mechanical or photocopying, recording, or otherwise without the prior permission of the publisher.

Published by

Charles University in Prague, Center for Economic Research and Graduate Education (CERGE) and

Economics Institute of the CAS, v. v. i. (EI)

CERGE-El, Politických vězňů 7, 11121 Prague 1, tel.: +420 224005 153, Czech Republic.

Printed by CERGE-EI, Prague

Subscription: CERGE-EI homepage: http://www.cerge-ei.cz

Phone: + 420224005153

Email: office@cerge-ei.cz

Web: http://www.cerge-ei.cz

Editor: Jan Zápal

The paper is available online at http://www.cerge-ei.cz/publications/working_papers/.

ISBN 978-80-7343-378-9 (Univerzita Karlova v Praze, Centrum pro ekonomický výzkum a doktorské studium)

ISBN 978-80-7344-394-8 (Národohospodářský ústav AV ČR, v. v. i.) 
CERGE-EI

P.O.BOX 882

Politických vězňů 7

11121 Praha 1

Czech Republic http://www.cerge-ei.cz 